\title{
Theoretical Modeling of QCM-D Sensors in Environmental Applications
}

\author{
M. V. Voinova, A. Vikström \\ Chalmers University of Technology, Fysikgårgen 1, 41296 Gothenburg Sweden, \\ marina.voinova@chalmers.se
}

\begin{abstract}
Theoretical work on QCM-D based acoustic sensors operated in gases, vapors and liquids containing dispersed microscopic particles is developed. With the main goal to provide theoretical support to the sensors for air pollution control, our analytical results supplemented with numerical calculations provide a way to quantify measurements taking into account the shear viscosity of liquid as a function of pollutant particles number for the small volume fractions (i.e., within the Einstein relation) and beyond the Einstein relation numbers. For the higher concentrations, the packing factor variation has been tested for modeling microscopic particles - in - water dispersions. Various types of pollutants with known density were used for modeling such as polymer particles, carbon black, fullerenes, mineral dust and bacteria. For the non-spherical particles, the shape factor was introduced as an additional model parameter. The role of softness of the surface-attached layer is studied in details.
\end{abstract}

Key words: QCM-D, acoustic sensors, environmental pollution control, particles, viscosity measurements, soft layer

\section{Background}

Bacterial, microbial and fungal contamination sprays brought at long distances with strong winds even over the ocean [1]. Microscopic sand from deserts, ash particles in the air and mineral dust of various nature is another example. Rain microdroplets, urban aerosols and polluted air coming from vehicle exhausts [2], industrial areas and pollen microparticles are common factors causing the asthma and other health problems among the allergic people. Weather conditions are not possible to control however it is possible to monitor the risk factors level with sensors. Microplastic pollution due to the rivers litter brought to the lakes and seas and then tired by waves, is a new type of contamination environment [3] invisible by eye but coming to our organism through the food chains (fish). The key question is how to increase the sensitivity of devices to the presence of micro- and nanoparticles in liquids, as well in vapors, aerosols and other liquidcontaining air components.

The environmental control of air pollution with BAW (bulk acoustic waves) sensors is a reliable and non-costly technological solution $[4,5]$. The QCM with the dissipation monitoring (QCM-D) is a widely used BAW-type of acoustic sensor for both air (gas) and liquid operating media. Two characteristics of the device, the resonance frequency shift, $\Delta f$, and the dissipation factor change, $\Delta \mathrm{D}$, measured simultaneously allow researchers to determine mechanical parameters of the layer adsorbed from air or liquid onto the surface of quartz resonator. The surface mass $m=\rho h$, a product of density and the thickness of the layer, as well as the product of shear viscosity and density, $\eta \rho$, can be estimated from measured shift in the resonance frequency and the dissipation. However, the presence of microparticles in liquid changes the response of the resonators.

The aim of the present work is to provide the theoretical support to the QCM experimental research in the air, water and soil quality control applications.

\section{Theory}

We model BAWs propagation in the colloidal dispersion of microscopic particles in water at low and high volume concentrations in terms of viscosity and density of colloidal solution. In our work we perform numerical calculations of the resonance frequency and the dissipation factor shift of the QCM oscillated in a complex fluid. The fluid is modeled as water dispersion of microscopic particles of different materials and shapes. The computer calculations are based on the theoretical analysis developed earlier (see for review [5]). The role of softness of guiding layer has been studied in more details presented in a separate subsection. 


\section{The model}

We model a quartz oscillator loaded with two homogeneous layers - a guiding polymer film under colloidal dispersion on the top (water with nanoparticles, NPs) considered as acoustically bulk medium of thickness $\mathrm{H}>>$, where $\delta=$ $\sqrt{ }(2 \eta / \rho \omega)$ is the viscous length. The colloidal dispersions are modeled as complex fluids of viscosity $\eta$ depending on nanoparticles volume fraction $\phi$, their shape and packing. Within the effective medium theory [6], the viscosity of the concentrated water colloid is given by the relation:

$$
\eta(\phi)=\eta_{W} \exp \left(\frac{\alpha \phi}{1-\kappa \phi}\right)
$$

In there, $\alpha$ is the so-called shape factor and $\mathrm{k}$ is the packing factor $[6,7]$. In the calculations, we used the expression (1) for the shear viscosity of the tested solution (water dispersion of nanoparticles of different density $\rho$ and shape). The averaged density for the dispersion can be written as follows:

$$
<\rho>=\left(\rho_{P}-\rho_{W}\right) \phi+\rho_{W}
$$

Here indices ' $W$ ' and ' $P$ ' denote water and particles, respectively. For the spherical particles in the low concentration limit (i.e., for the volume fraction $\phi$ less than $20 \%$ ), the Einstein relation is valid [6]:

$$
\eta \approx \eta_{W}(1+2.5 \phi)
$$

\section{The results}

By using the general expression for the acoustic impedance obtained earlier (see in [5]), together with formulae $(1,2)$, we calculated the $\Delta f(\phi)$ and $\Delta D(\phi)$ characteristics.
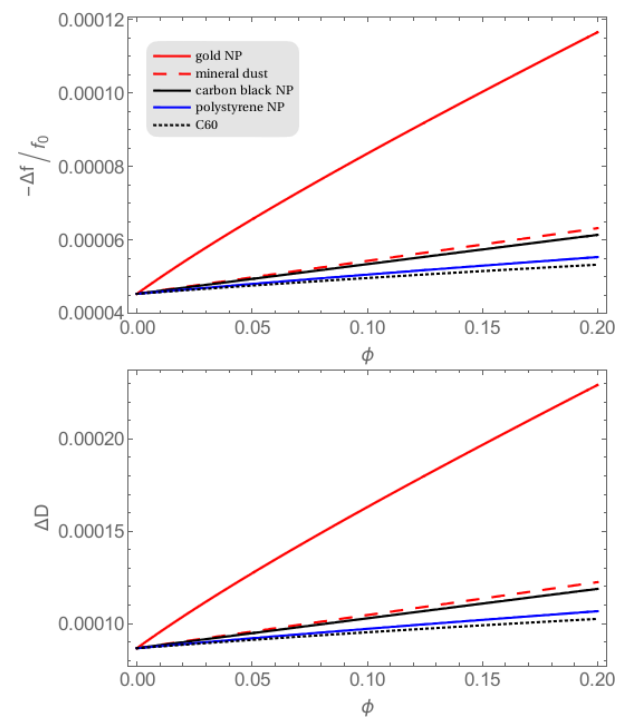

Fig. 1. Upper graph: the resonance frequency changes $\Delta f$ vs $\phi$ calculated for the different materials. Bottom graph: the dissipation changes $\Delta D$ vs $\phi$.
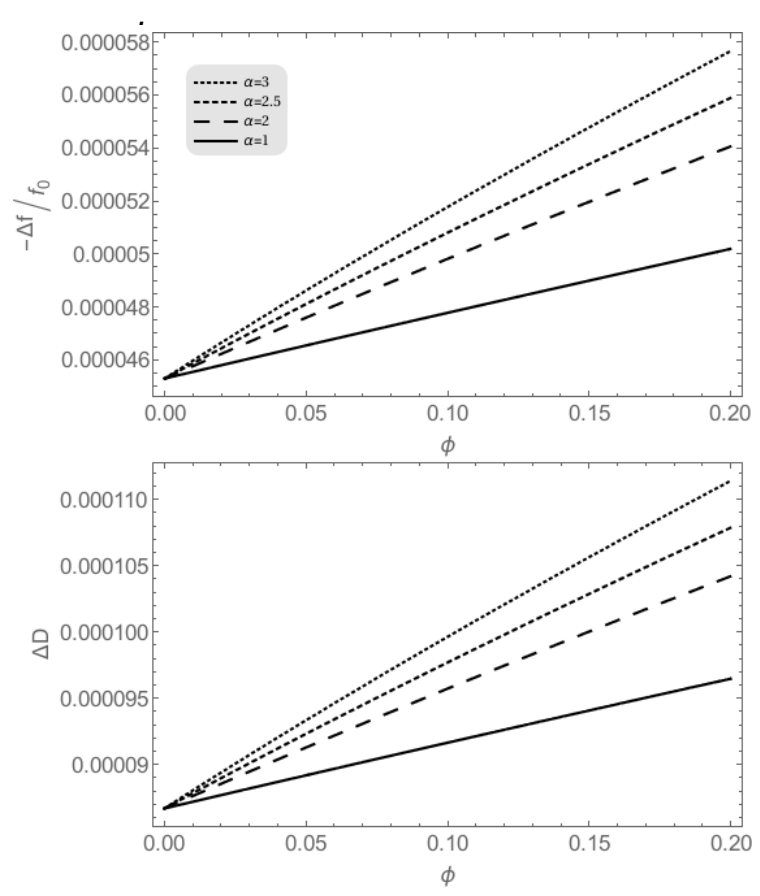

Fig. 2. Upper graph: calculated resonance frequency shift $\Delta f$ vs $\phi$. Bottom graph: the dissipation changes vs $\phi$. The characteristics were calculated for the different shape factor values. Pollutant: EColi.
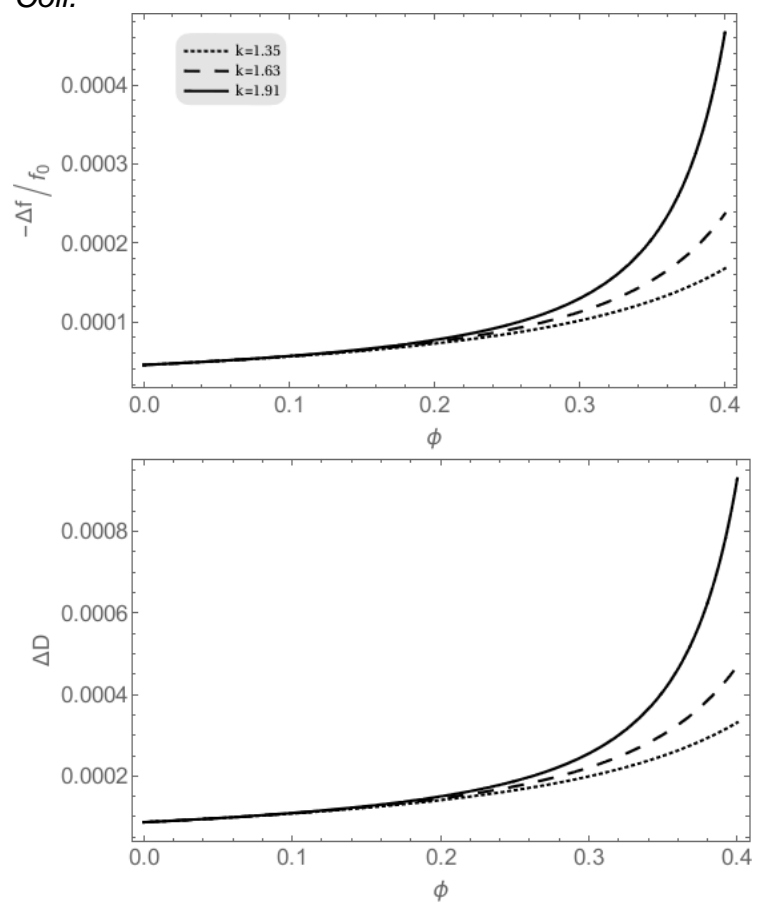

Fig. 3. Upper graph: the resonance frequency changes $\Delta f$ vs $\phi ;$ Bottom graph: the dissipation changes $\Delta D$ vs $\phi$. The characteristics were calculated for the different packing factor $k$ values. Pollutant: mineral dust.

Fig. 1 shows QCM characteristics calculated for the different composition of water colloids by varying the material of particles - gold NPs, mineral dust, carbon black, C60, and polymer 

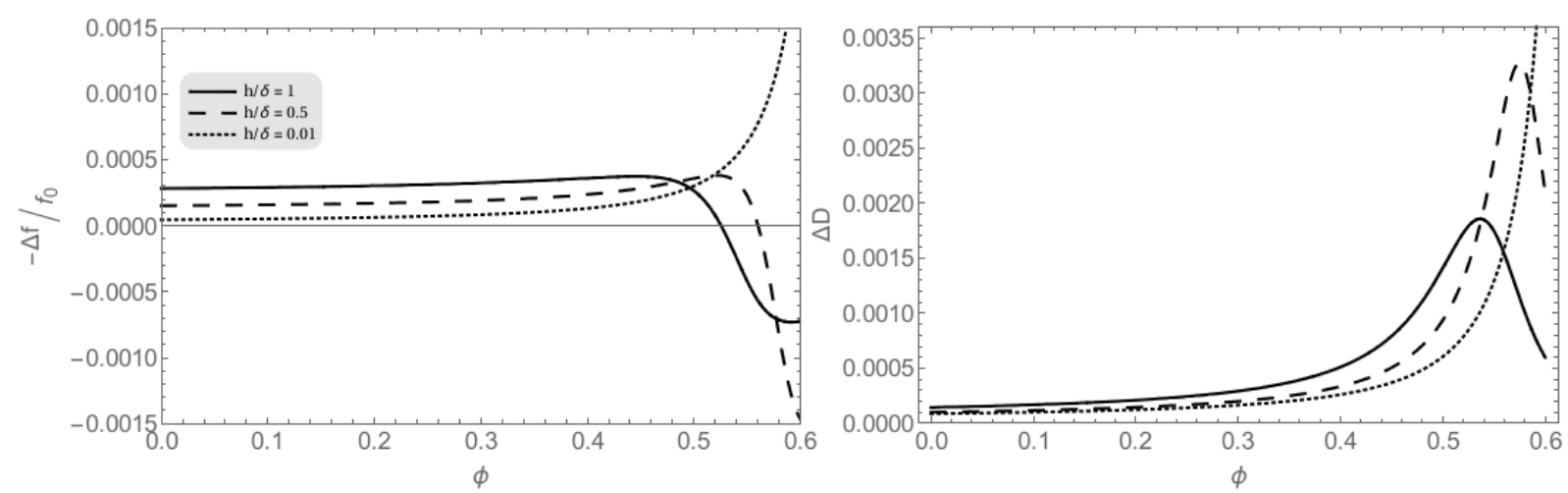

Fig. 4. To the left: Calculated frequency shift $\Delta f / f_{0}$ vs volume fraction of NPs in water. To the right: Calculated dissipation vs volume fraction of NPs (polysterene) in water. The plots illustrate the case of soft polymer film as a guiding layer (PGA-PLL= polyl(L-lysine)-poly (L-glutamic acid) material parameters were estimated from [8]; mass density $=1500 \mathrm{~kg} / \mathrm{m}^{3}$, shear viscosity $=3.4 \mathrm{mPa}$ sec, shear modulus $=150 \mathrm{kPa}, f_{0}=1 \mathrm{MHz}, \delta=0.85 \mathrm{micron}$ ).

spherical particles within the Einstein relation ( $\alpha$ $=2.5, \phi<0.2)$. The densities of the particles material are given in Table 1. For non-spherical particles, the shape factor $\alpha$ variations (between 1 and 3) have been introduced (Fig. 2). Since both acoustical characteristics of the top fluid depend on the square root of the product of shear viscosity and density of fluid,

$$
\Delta f \propto \sqrt{\eta \cdot \rho}, \Delta D \propto \sqrt{\eta \cdot \rho}
$$

for the dilute dispersions $(0<\phi<0.2)$ the response is nearly linear (see Fig. 1 and Fig. $2)$. When the volume fraction of particles exceeds 20\%, both $\Delta \mathrm{f}(\phi)$ and $\Delta \mathrm{D}(\phi)$ characteristics rapidly grow. For the concentrated dispersions with $0.2<\phi<0.4$, one can introduce the packing factor $\mathrm{K}$ as an additional model parameter (Fig. 3). In all the plots in Figs.1-3, the fundamental resonance frequency is taken $1 \mathrm{MHz}$, the PMMA film is considered as a rigid guiding layer.

\section{Soft layer under colloid dispersion}

Then, the soft layer of varying thickness $h$ has been considered as a mass guiding layer.

$$
\begin{aligned}
& \Delta f \approx-\frac{\sqrt{\pi \rho \eta f_{0}}}{2 \pi m_{q}}-\frac{h_{1} \rho_{1} f_{0}}{m_{q}}\left\{1-\frac{\rho \eta}{\rho_{1} \eta_{1}} \frac{\tan ^{2} \vartheta}{1+\tan ^{2} \vartheta}\right\} \\
& \Delta D \approx \frac{1}{m_{q}} \sqrt{\frac{\rho \eta}{\pi f_{0}}}+\frac{2 h_{1} \rho \tan \vartheta}{m_{q}} \frac{1}{1+\tan ^{2} \vartheta}
\end{aligned}
$$

$\tan \vartheta \equiv G_{1}^{\prime \prime} / G_{1}^{\prime}$.

For the acoustically thin viscoelastic solid film, the analytical expressions for the $\Delta f$ and $\Delta D$ can be obtained (4-6). The softness of the film material here is given by the loss tangent, $\tan \vartheta$. For the thicker films, the computer modeling has been used. The results shown on Fig. 4 demonstrate the deviation from linear behavior for both $\Delta f$ and $\Delta \mathrm{D}$ at the volume fraction values larger than approximately 0.45 , and showing peaks in the dissipation factor $\Delta \mathrm{D}(\phi)$.

Tab.1: Density of particle material used in the calculations (1, 5, 6 from [14]).

\begin{tabular}{|c|c|c|}
\hline Number & $\begin{array}{c}\text { Type of } \\
\text { material }\end{array}$ & $\begin{array}{c}\text { Density in } \\
\text { bulk, } \mathrm{kg} / \mathrm{m}^{3}\end{array}$ \\
\hline 1 & Gold particles & 19300 \\
\hline 2 & $\begin{array}{c}\text { Mineral dust } \\
\text { (Fine sand) }\end{array}$ & 2650 \\
\hline 3 & Carbon black & 2260 \\
\hline 4 & C60 & 665 \\
\hline 5 & E-Coli & 1160 \\
\hline 6 & Polystyrene & 1050 \\
\hline
\end{tabular}

One should notice that for the thicker soft films (for example, for $h / \delta=0.5$, comparing to the thin film case $(h / \delta=0.01)$, shown as a dotted line in Fig. 4), the resonance frequency shift diminishes instead of growing up with the increased volume fraction of NPs in water. At some relationship between the viscosity of the top fluid (i.e., at some particular volume fraction $\phi$ of NPs) and the viscosity/elasticity of the underlying soft layer, the resonance frequency shift changing sign becoming positive. This finding should be studied experimentally with attention since in many practical situations, e.g. in sensors working in humid/wet conditions and biosensors in liquid applications the surface layer is soft (viscoelastic). The soft layer could be a polymer guiding film or adsorbed layer formed at particles adhered to the surface of the quartz oscillator (if the surface adsorption of the colloid constituents is not prevented somehow).

\section{Discussion}

In our choice of the pollutant materials listed in the Table 1, we were motivated by the following 
experimental findings. Recently, comparative studies for the plastic NPs monitored in dry and humid conditions including the ordering of NPs phenomenon were reported in [8]. Another "hot spot' is the carbon black coming from combustion particles [2] and fullerenes (C60 NPs) appeared in the industrial areas of cities [9]. The analysis of fullerene samples [9] shows more than $50 \%$ of 660 volume fraction in soil collected in the particular areas. The understanding of interaction of fullerenes with water in wet biological samples is an important part of the environmental health research [9]. In particular, it was shown [10] that the grass pollen particles may bind to diesel nanoparticles causing allergy and asthma diseases. The usage of the QCM sensor coated with the pollen-imprinted polymer film [12] for the allergen analysis opened a way to study the particles micro-rheology. Finally, a remarkable effort is measurement of water presence in oil [13]. The authors deduce that water droplets sticking to the surface of oscillator may be a reason of the highly non-linear response of the micro-acoustic sensor device. However, it was suggested [13] to employ this deviation from linearity as beneficial, for example, for the control of the apparent water contamination of oily liquids (such as engine and lubrication oils) essential for the diagnostics and environmental oil condition monitoring.

\section{Conclusions}

The study of density-viscosity of complex fluids with the help of QCM may bring new questions in the interpretation of results when the characteristics of device are non-linear. The theoretical formulation and numerical analysis reported in the present work provide quantitative explanation for the effects arising due to the variation in the volume fraction of NPs in water and accounting for the softness of the guiding layer of the sensor.

\section{Acknowledgements}

To EuNetAir Action colleagues for the fruitful discussions and to EC for the financial support of scientific COST Action TD1105 meetings where our results were reported.

\section{References}

[1] C .A. Kellogg, D. W. Griffin, Aerobiology and the Global Transport of Desert Dust, Trends in Ecology and Evolution 21, (2006); doi:10.1016/j.tree.2006.07.004.

[2] A. Kocbach, Y. Li, K.E. Yttri, F. R Cassee, P. E. Schwarze, E. Namork, Physicochemical Characterisation of Combustion Particles from Vehicle Exhaust and Residential Wood Smoke,
Particle and Fibre Toxicology 3, 1-10 (2006); doi:10.1186/1743-8977-3-1.

[3] M. Colea, P. Lindequea, C. Halsband, T. S. Galloway, Microplastics as Contaminants in the Marine Environment: A Review, Marine Pollution Bulletin 62, 2588-2597 (2011); doi:10.1016/j.marpolbul.2011.09.025.

[4] D. Johannsmann, The Quartz Crystal Microbalance in Soft Matter Research. Fundamentals and Modeling, Springer, 2015.

[5] M.V.Voinova, M.Jonson, The Quartz Crystal Microbalance, Chapter 9. In: Chemical sensors. Comprehensive sensor technologies. V.4: Solidstate devices. Ed. G. Korotcenkov. Momentum Press, LLC, New York, 2011.

[6] M.J. Mooney, The Viscosity of a Concentrated Suspension of Spherical Particles, Journal of Colloidal Science 6, 162-170 (1951).

[7] K. Takamura, T.G. van de Ven, Comparisons of Modified Effective Medium Theory with Experimental Data on Shear Thinning of Concentrated Latex Dispersions, Journal of Rheology 54, 1-16 (2010);doi:10.1122/1.3263700

[8] J.A. Phelps, S. Morisse, M. Hindie, M.-C. Degat, E. Pauthe, P. R. Van Tassel, Nanofilm Biomaterials: Localized Cross - Linking To Optimize Mechanical Rigidity and Bioactivity, Langmuir 27, 1123-1130 (2011);doi: 10.1021/la104156c.

[9] J. M. Hankett, W. R. Collin, P. Yang, Z. Chen, M. Duhaime, Low-Volatility Model Demonstrates Humidity Affects Environmental Toxin Deposition on Plastics at a Molecular Level, Environmental Science \& Technology 50, 1304-1312 (2016); doi: 10.1021/acs.est.5b05598.

[10] A. Carboni, R. Helmus, E. Emke, N. van den Brink, J. R. Parsons, K. Kalbitz, P.de Voogt, Analysis of Fullerenes in Soils Samples Collected in The Netherlands, Environmental Pollution 219, 47-55 (2016) ; doi:10.1016/j.envpol.2016.09.034.

[11] R. B. Knox, C. Suphioglu, P. Taylor, R. Desat, H. C. Watson, J. L. Peng, L. A. Bursill, Major Grass Pollen Allergen Lol p1 Binds to Diesel Exhaust Particles: Implications for Asthma and Air Pollution, Clinical and Experimental Allergy 27, 246-251(1997);doi: 10.1111/j.13652222.1997.tb00702.x.

[12] M. Jenik, A. Seifner, P. Lieberzeit, F.L. Dickert, Pollen-Imprinted Polyurethanes for QCM Allergen Sensors, Analytical and Bioanalytical Chemistry 394, 525-8 (2009); doi: 10.1007/s00216-0092718-8.

[13] B. Jakoby, M.J. Vellekoop, Physical sensors for water-in-oil emulsions, Sensors and Actuators A 110, 28-32 (2004); doi:10.1016/j.sna.2003.08.005

[14] M.Godin, A.K.Bryan, T.P.Burg, Measuring the Mass, Density and Size of Particles and Cells Using a Suspended Microchannel Resonator, Applied Physics Letters 91, 123121 (2007); doi: 10.1063/1.2789694. 